\title{
Revisiting Julg's Structural Approach to Aromaticity
}

\author{
Myriam S. de Giambiagi, Mario Giambiagi, and Aloysio Paiva de Figueiredo \\ Centro Brasileiro de Pesquisas Físicas, Rua Dr. Xavier Sigaud 150, \\ 22290-180 Rio de Janeiro, RJ, Brasil \\ Reprint requests to Prof. M. S. G.; Fax: 5521-5867400; E-mail: rio@cbpf.br \\ Z. Naturforsch. 56 a, 413-415 (2001); received January 16, 2001

\begin{abstract}
Julg's classical formula for aromaticity is updated so as to involve bond indices. A simple $\mathrm{CNDO} / 2$ calculation is shown to account satisfactorily for heterocyclic typical rings and other miscellaneous systems. Results are compared with a multicenter MO bond index recently introduced as an aromaticity measure.
\end{abstract}

Key words: Aromaticity; Julg's Formula; Multicenter MO Bond Index.

Possibly the only unanimity about the classical concept of aromaticity is that it is far from being a well defined quantity [1]. Despite it has been the subject of ferocious criticism about 30 years ago, these last years have witnessed a revival of the term, and several kinds of approaches have been and are being proposed to the notion of aromaticity, see e. g. [2,3]. One of the facets of aromaticity has been put forward in a formula by Julg and François [4], modified later [5]. Their appealing viewpoint focuses on the structural criterion for aromaticity, namely the uniformity of interatomic distances [4] and electronic density [5]. The first one, applied to hydrocarbons, is accounted for as [4]

$$
A_{1}^{\prime}=1-(225 / n) \sum_{(r s)}\left(1-d_{r s} / d\right)^{2}
$$

where $n$ is the number of peripheral bonds $(r s), d_{r s}$ their lengths and $d$ the mean length. In order to apply the formula to heterocyclic molecules, $A_{1}^{\prime}$ is multiplied [5] by

$$
A_{2}^{\prime}=\prod_{(i j)}\left[1-\left(\Delta q_{i j} / d_{i j}\right)^{2}\right],
$$

where $\Delta q_{i j}$, the charge gradient, takes into account the resistance opposed to the electronic circulation. Aromaticity $A^{\prime}$ is thus $A_{1}^{\prime} \bullet A_{2}^{\prime}$. This approximation proved useful in estimates for hydrocarbons and a few heterocycles [1, p. 49].

We ourselves have proposed an MO (molecular orbital) multicenter bond index as a measure of ring aromaticity [6]. We have applied it to a wide variety of systems, and it has proven to perform satisfactorily in most of them, being in good agreement with other indices in the literature and easy to implement. For five-membered (5-m) rings, however, the results suffer from drawbacks which we left as open questions. We intend to face them here and study some other systems.

It has been remarked [7] that different types of bonds can have the same length and yet be quite different in nature, such as $\mathrm{C}-\mathrm{C}, \mathrm{C}-\mathrm{N}$ or $\mathrm{C}-\mathrm{O}$; hence, it would be better to consider bond orders rather than bond lengths. In [4], $\pi$ bond orders have been used to estimate bond distances, but bond orders for different types of atoms are subject to the same objection.

Several years ago, we generalized the Wiberg bond index [8] to non-orthogonal bases, through the first order density matrix $2 \prod$. For closed shells, the index $I_{A B}$ for the bond between atoms $\mathrm{A}$ and $\mathrm{B}$ is $[9,10]$

$I_{A B}=4 \sum_{a \in A, b \in B} \prod_{a}^{b} \prod_{b}^{a} ; q_{A}=\left(I_{A A}+\sum_{B \neq A} I_{A B}\right) / 2,(3)$

where

$$
\prod_{a}^{b}=\sum_{i} x_{i a} x^{i b}
$$

and $x_{i a}\left(x^{i b}\right)$ are the covariant (contravariant) MO coefficients and $i$ runs over the occupied MO levels. The charge is Mulliken's gross population with a quite different partition into self-charge $\left(I_{A A}\right)$ and active charge, $I_{A B}$ being related to exchange correlation and comparable between different types of atoms 
$[6,9,10] . I_{A B}$ is generalized for multicenter bonds as below [11]; for the calculation of $I_{\text {ring }}$ [6] we have used

$$
I_{A B \cdots L}=2^{L} \sum_{a \in A, b \in B, \cdots, \ell \in L} \prod_{a}^{b} \prod_{b}^{c} \cdots \prod_{\ell}^{a} .
$$

We have thus found interesting to revisit Julg's proposal for aromaticity in the light of our formulations. $I_{A B}$ is inversely proportional to the bond length, being equal to 1 for a purely single bond and 2 for a double bond. If, as before, $A=A_{1} \bullet A_{2}, A_{1}$ and $A_{2}$ shall be for us

$$
\begin{aligned}
& A_{1}=1-(6.4 / n) \sum_{(A B)}\left(1-\bar{I} / I_{A B}\right)^{2} ; \\
& A_{2}=\left[\prod_{(C D)}\left[1-\left(\Delta q_{C D} I_{C D}\right)^{2}\right]^{1 / n^{\prime}} .\right.
\end{aligned}
$$

The constant 6.4 has been obtained, similarly to Julg [4], such as to yield $A_{1}=1$ for benzene and $A_{1}=0$ for one Kekulé structure, where $I_{A B}$ is alternatively 1 and 2 . Incidentally, rectangular cyclobutadiene also yields $A_{1}=0$; in both cases $\bar{I}=1$.5. Just as (6) (or (1)) involves the arithmetic average of $I$ (or $d$ ), we have chosen to add for $A_{2}$ the geometrical average of the product involved; $n^{\prime}$ is the number of terms in this product.

In the context behind formulae such as Julg's, or the expressions which we are proposing, it would be clearly inconsistent to use an $a b$ initio calculation method. It has been recently been noted that, despite the spectacular advances in computational chemistry, the use of semiempirical methods is actually increasing rather than decreasing; see [12] for the discussion. Among them, PM3 and AM1 of the MOPAC package are possibly the most used at present [12]. Our experience has indicated that, although both of them are adequate for estimating bond indices, the electronic density is unsatisfactory. We have therefore chosen to calculate $q_{A}$ and $I_{A B}$ in the $\mathrm{CNDO} / 2$ approximation, running before an AM1 geometry optimization as we did in our previous work, where indices were calculated within PM3 [6].

CNDO is seen in the literature mainly in its parameterized version for spectroscopical applications CNDO/S [13], but many references may still be found for CNDO/2 applications; see, e. g. [14, 15].

The trends in the $A$ values corresponding to the rings in naphthalene, anthracene, phenanthrene and
Table 1. Aromaticity for systems consisting of typical 6-c and 5-c rings.

\begin{tabular}{|c|c|c|c|c|}
\hline & & $A$ & $I_{\text {ring }}$ & $I_{\text {ring }}^{\pi}$ \\
\hline 1 & $\triangle$ & 1 & 0.0163 & 0.0050 \\
\hline 2 & $\Delta$ & 0.9590 & 0.0148 & 0.0040 \\
\hline 3 & $\stackrel{O}{\triangle}$ & 0.9227 & 0.0211 & 0.0037 \\
\hline 4 & $\stackrel{S}{S}$ & 0.9832 & 0.0691 & \\
\hline 5 & U & 0.5852 & 0.0135 & 0.0126 \\
\hline 6 & $\Delta$ & 0.4943 & 0.0254 & 0.0230 \\
\hline
\end{tabular}

\begin{tabular}{l|ll}
\hline & $A$ & $I_{\text {ring }}[6]$ \\
\hline benzene & 1 & 0.0883 \\
pyridine & 0.9408 & 0.0877 \\
pyridazine & 0.9826 & 0.0878 \\
pyrazine & 0.9539 & 0.0878 \\
pyrimidine & 0.8250 & 0.0864 \\
$1,3,5$ triazine & 0.6627 & 0.0840 \\
pyrrole & 0.8591 & 0.0962 \\
furan & 0.6514 & 0.0541 \\
thiophene & 0.8620 & 0.0696 \\
\hline
\end{tabular}

Table 2. Aromaticity for miscellaneous molecules.

1: cyclopropane; 2: aziridine; 3: oxirane; 4: thiirane; 5: dimethylenecyclobutene; 6 : fulvene

azulene agree qualitatively with those of $I_{\text {ring. The }}$ molecular $A$ values are respectively $0.9171,0.8877$, 0.9152 , and 0.8456 , which are satisfactory from the chemical viewpoint [4, 5]. Julg's formula was devised for $\pi$ calculations, while (6) embodies the $\sigma$ framework. Anyway, no large differences are found for hydrocarbons; they may instead be more significant for heterocycles.

We show in Table 1 the $A$ results for benzene and typical monocyclic azines, as well as the most common 5-c ring heterocyclic compounds; we have added our previous $I_{\text {ring }}$ values [6]. The $A$ values manifest a greater discrimination between azines. The behaviour for the 5-c rings corrects the flaw of $I_{\text {ring }}$ for these systems (the same flaw is shared by other aromaticity indices [6]), bringing them below the benzene value.

We have calculated a few miscellaneous molecules with interesting results for $A$, shown in Table 2, together with $I_{\text {ring }}$.

Any hydrocarbon consisting of a single regular polygon shall have $A=1$ following (5); so does cyclopropane. Nevertheless, as it is considered the paradigm of $\sigma$ aromaticity [1, chap. 7], we shall discuss some peculiar features of this molecule. 
Cyclopropane has been described as a system with a central two-electron three-center bond ("super $\sigma$ bond") and two peripheral four-electron three-center bonds (" $\pi$ bonds") [1 (p. 260), 16]. Actually, this description refers to radial and tangential orbitals rather than conventional $\sigma$ and $\pi$ ones. Now, we have analysed this kind of model from a quite different standpoint, more in the spirit of this approach [17].

The electron count which we have proposed for the three-center bond $A B C$ is [17]

$$
\chi=I_{A B}+I_{B C}+I_{A C},
$$

for the self-charge is confined within the atoms. For cyclopropane, $I_{A B}$ is 0.9893 , of which 0.0291 is $\pi$. Thus, in these terms, we would say that we have a three-center $\sigma$ bond with 2.88 electrons and a threecenter $\pi$ bond with only 0.09 electrons. This amounts to our description of $\sigma$ aromaticity in cyclopropane and is consistent with our results for 3-c indices. We have $I_{123}=0.0163$; unlike what happens for 6-c and 5-c rings [18], this is mostly $\sigma$, the $\pi$ contribution being 0.0050 .

The geometries obtained through the AM1 optimization (which we have not reproduced here) agree with those appearing in the literature as results from much more elaborated methods. The adequacy of the adopted procedure is thus confirmed.

For the other triangular systems aromaticity is also mainly $\sigma$. The $A$ values for the sequence aziridine, oxirane, thiirane of Table 2 show a trend closely similar to that of the sequence pyrrole, furan, thiophene of Table 1. This is not verified for the corresponding $I_{\text {ring }}$ values.

Dimethylenecyclobutene (DMCB) and fulvene have been calculated in a recent modern VB study using spin-coupled theory with $6-31 \mathrm{G}^{* *}$ basis [19]; the conclusion is that resonance energies for both systems are very low. The $I_{\text {ring }}$ values (almost wholly $\pi$ ) are similar to the above ones in the table, while the $A$ values are decidedly lower.

For fulvene, it is possible to calculate $A$ including the exocyclic bond which a priori may join the conjugation; in this way, a value of 0.4752 is obtained. In this case, the calculation of $I$ does not allow an estimate including the exocyclic bond.

There is general consensus about taking benzene as a reference whenever aromaticity is mentioned, independently from the measure chosen for this magnitude. Now then, benzene has unique features within the $\pi$ electron framework, as its high symmetry determines by itself the MO's, aside from normalization. We have shown [18] that $I_{\text {ring }}$ for benzene, being mainly $\pi$, is almost known a priori; this gives a sound basis for the reference role ascribed to the molecule in aromaticity.

We can conclude, from what was discussed above, that Julg's updated formula such as proposed here deserves still to be considered as an aromaticity index related to the structural criterion [1].
[1] V. I. Minkin, M. N. Glukhovtsev, and B. Y. Simkin, Aromaticity and Antiaromaticity: Electronic and Structural Aspects, Wiley \& Sons, New York 1994.

[2] D. B. Chesnut and L. J. Bartolotti, Chem. Phys. 253, 1 (2000).

[3] Z. H. Yu, Z.-Q. Xuan, T.-X. Wang, and H.-M. Yu, J. Phys. Chem. A 104, 1736 (2000).

[4] A. Julg and P. François, Theoret. Chim. Acta 8, 249 (1967).

[5] A. Julg in E. D. Bergmann and B. Pullman (Eds.), Aromaticity, Pseudo-Aromaticity, Anti-Aromaticity, The Israel Academy of Sciences and Humanities, Jerusalem 1971, p. 383.

[6] M. Giambiagi, M. S. de Giambiagi, C. D. dos Santos Silva, and A. Paiva de Figueiredo, Phys. Chem. Chem. Phys. 2, 3381 (2000).

[7] A. R. Katritzky, P. Barczynski, G. Musumarra, D. Pisano, and M. Szafran, J. Amer. Chem. Soc. 111, 7 (1989).

[8] K. Wiberg, Tetrahedron 24, 1083 (1968).

[9] M. Giambiagi, M. S. de Giambiagi, D. R. Grempel, and C. D. Heymann, J. Chim. Phys. 72, 15 (1975).
[10] M. S. de Giambiagi, M. Giambiagi, and F. E. Jorge, Z. Naturforsch. 39 a, 1259 (1984).

[11] M. Giambiagi, M. S. de Giambiagi, and K. C. Mundim, Struct. Chem. 1, 423 (1990).

[12] A. A. Bliznyuk and A. P. Rendell, J. Comput. Chem. 20, 629 (1999).

[13] J. Fleischhauer, A. Koslowski, C. Repges, K.-P. Gulden, and G. Bringmann, Z. Naturforsch. 53 a, 993 (1998).

[14] S. N. Datta, V. Nehra, and A. Jha, J. Phys. Chem. B 103, 8768 (1999).

[15] D. P. Ojha, Mol. Mater. 12, 377 (2000).

[16] D. Cremer, Tetrahedron 44, 7421 (1988) and references therein.

[17] M. S. de Giambiagi, M. Giambiagi, and J. Herrera, Z. Naturforsch. 49 a, 754 (1994).

[18] C. G. Bollini, M. Giambiagi, M. S. de Giambiagi, and A. P. de Figueiredo, J. Math. Chem. 28, 71 (2001).

[19] J. M. Oliva, Int. J. Quantum Chem. 71, 319 (1999). 